\title{
From landrace to modern hybrid broccoli: the genomic and morphological domestication syndrome within a diverse $B$. oleracea collection
}

\author{
Zachary Stansell (1) ${ }^{1,2}$ and Thomas Björkman ${ }^{1,2}$
}

\begin{abstract}
Worldwide, broccoli (Brassica oleracea var. italica) is among the most economically important, nutritionally rich, and widely-grown vegetable crops. To explore the genomic basis of the dramatic changes in broccoli morphology in the last century, we evaluated 109 broccoli or broccoli/cauliflower intermediates for 24 horticultural traits. Genotype-bysequencing markers were used to determine four subpopulations within italica: Calabrese broccoli landraces and hybrids, sprouting broccoli, and violet cauliflower, and to evaluate between and within group relatedness and diversity. While overall horticultural quality and harvest index of improved hybrid broccoli germplasm has increased by year of cultivar release, this improvement has been accompanied by a considerable reduction in allelic diversity when compared to the larger pool of germplasm. Two landraces are the most likely founding source of modern broccoli hybrids, and within these modern hybrids, we identified 13 reduction-in-diversity genomic regions, 53 selective sweeps, and 30 (>1 Mbp) runs of homozygosity. Landrace accessions collected in southern Italy contained 4.8-fold greater unique alleles per accessions compared to modern hybrids and provide a valuable resource in subsequent improvement efforts. This work broadens the understanding of broccoli germplasm, informs conservation efforts, and enables breeding for complex quality traits and regionally adapted cultivars.
\end{abstract}

\section{Introduction}

Broccoli (Brassica oleracea var. italica) and cauliflower (B. oleracea var. botrytis) are the most widely-grown brassica vegetable crops internationally, with a cumulative production area of 1.4 million $\mathrm{Ha}^{1}$. $\mathrm{F}_{1}$ hybrid broccoli is the most economically important brassica vegetable crop in the United States with a farm-gate value of $\sim 1$ billion $\mathrm{USD}^{2}$.

In recent years, considerable progress understanding the $B$. oleracea crop group has been made. Specifically, several key objectives have been accomplished: parsing fundamental genomic architecture $^{3-5}$, publication of high-quality reference genomes ${ }^{6-8}$, evaluating diversity and domestication processes ${ }^{9-21}$, and identifying genomic

Correspondence: Zachary Stansell (zjs29@cornell.edu)

'Cornell University, School of Integrative Plant Science, Cornell University, Ithaca, NY 14850, USA

${ }^{2}$ Cornell AgriTech, Cornell University, Geneva, NY 14456, USA regions or candidate genes associated with horticultural quality $^{22-27}$ and biotic/abiotic stress resistance ${ }^{28-35}$.

While modern broccoli cultivars are distinct from their landrace precursors in heading induction requirement, time to maturity, crown size and architecture, and secondary metabolic profile $14,17,36-44$, the basis for these dramatic changes remains largely unexplored using genomics era tools, such as genotype-by-sequencing. Here, we build on previous work by clarifying the relationship of elite broccoli germplasm within a larger pool of italica germplasm, and characterize the genomic and phenotypic changes that occurred during this improvement process ${ }^{16}$.

The italica cultivar group is a member of the $\mathbf{C C}$ genome B. oleracea $(2 \mathrm{n}=18)$ coenospecies and was domesticated from crop wild relatives in the Mediterranean Basin by human selection under local conditions, followed by improvement into landrace types in the central Mediterranean region, most likely within the southern Italic

\section{(c) The Author(s) 2020}

(c) (i) Open Access This article is licensed under a Creative Commons Attribution 4.0 International License, which permits use, sharing, adaptation, distribution and reproduction c. in any medium or format, as long as you give appropriate credit to the original author(s) and the source, provide a link to the Creative Commons license, and indicate if changes were made. The images or other third party material in this article are included in the article's Creative Commons license, unless indicated otherwise in a credit line to the material. If material is not included in the article's Creative Commons license and your intended use is not permitted by statutory regulation or exceeds the permitted use, you will need to obtain permission directly from the copyright holder. To view a copy of this license, visit http://creativecommons.org/licenses/by/4.0/. 
Peninsula and Sicily ${ }^{14,17,19,45-48}$. Italica domestication is complicated by emergence from a relatively large and admixed pool of landraces, consistent with a Vavilovian model of local assortment of morphologically and physiologically heterogeneous populations ${ }^{47,49}$.

Over time, Calabrese broccoli production and breeding has generally spread westward. Various italica and botrytis landraces from southern Italy were introduced to the United Kingdom during the 18th century ${ }^{39}$. Although Calabrese broccoli was initially brought to the United States by immigrants from southern Italy, it only gained popularity there post-WWII, following development of improved open-pollinated cultivars such as 'Waltham 29' (1950) from the Massachusetts Experiment Station ${ }^{50,51}$. Supported by American and Japanese breeding of commercially successful hybrids such as 'Premium Crop' (1975), 'Packman' (1983), and 'Marathon' (1985), production was shifted to the cooler valleys along the western U.S. coast allowing year-round production ${ }^{51}$. These hybrid breeding efforts increased yield (head size and harvest index), horticultural quality, regional adaptation, while decreasing days to complete growing cycle $^{39,51,52}$. China is now the largest producer of broccoli and Chinese cultivars appear to be derived from a core collection of Japanese germplasm, exhibiting close genetic relationships and reduced diversity ${ }^{20}$.

Traditionally, small farmers and gardeners in periurban farming environments have practiced in-situ preservation of diverse and locally adapted $B$. oleracea landraces via seed-saving and informal selection ${ }^{11,14,17,40,44,53-56}$. Italica landraces from Italy are more genetically diverse than other italica landraces ${ }^{43}$, and this diverse germplasm has been jeopardized, prompting ex-situ and in-situ conservation efforts and economic sustainability policies $^{14,17,38,40,44,57}$. Several landraces have been suggested as potential italica primitives: the highly branching 'Broccolo Nero' lacking apical dominance ${ }^{17}$, 'Mugnoli' from the Salento region ${ }^{14,58,59}$, and a Sicilian landrace, 'Cavolo Broccolo Calabrese Tardivo' that collocated with crop wild relatives ${ }^{43}$.

Several additional italica vegetables are known. The sprouting broccoli type is a distinct vegetable and is characterized by many lateral inflorescences, a small apical crown typically bisected by cauline leaves, later heading and flowering, and prized culinary properties.

Purple cauliflower types are most common in southern Italian regions and exhibit an intermediate italical botrytis phenotype, with leaf structures more similar to botrytis, intermediate developmental arrest stage and glucosinolate profiles, and variable curd coloring (e.g., purple, green, red, or white $)^{36,38}$. The tropical cauliflower type was first bred in $19^{\text {th }}$ century India and is now common in Southeast Asian markets, typically producing cauliflower-like heads above $22^{\circ} \mathrm{C}$ and broccoli-like heads below $16^{\circ} \mathrm{C}^{36,60,61}$. Violet and tropical cauliflower vegetables appear as italica/botrytis intermediates in morphologic and population structure analyses ${ }^{16,29,47}$. It is currently unclear if these intermediates exist as recent hybrids or are derived from an ancestral breeding pool. Additional italica or italical botrytis vegetables are most abundant in southern Italy and include the putative botanical classes Romanesco, Di Jesi, and Maceracta ${ }^{12,14,17,36}$.

To understand and document the improvement process of modern broccoli cultivars from within the larger pool of italica germplasm, 109 unique landrace and $F_{1}$ hybrid accessions were evaluated for 24 horticultural quality traits and 31,811 high quality genotype-by-sequencing markers were generated. We applied multiple selection scan methods to contrast a broad sampling of modern broccoli hybrids against a pool of broccoli landraces and identified patterns of population differentiation, regions of reduced diversity, selective-sweeps, runs of homozygosity, and developmental candidates within modern Calabrese broccoli hybrids. When compared with the larger pool of italica germplasm, genomic regions enriched in these signatures of crop improvement were considered as possible targets of human selection. This works clarifies the relationship of modern broccoli within $B$. oleracea var. italica, describes phenotypic changes that have occurred during improvement, and prepares the foundation for genomics-enabled broccoli improvement.

\section{Results}

Four inferred subpopulations were determined via analysis of genotype-by-sequencing markers of all accessions $(N=109$; Fig. 1; Supplementary Fig. 1): Calabrese hybrids $(N=53)$, Calabrese landraces $(N=28)$, sprouting broccoli $(N=18)$ and violet cauliflower $(N=10)$, and subpopulation membership was highly concordant with phenotypic evaluations within replicated field trials.

\section{Genotyping}

To distinguish among closely related genotypes and understand genomic variation, we conducted genotypeby-sequencing of all evaluated accessions, producing $1,185,484,626$ raw reads, with over $88 \%$ of bases surpassing Q30. The raw reads generated 10,108,099 tags producing 247,220 aligned SNPs. After filtering, 31,811 high quality SNPs were retained for further analysis. Averaged across these SNPs, site missingness and heterozygosity was $3.1 \%$ and $15.9 \%$, and minor allele frequency was $12.8 \%$ (Fig. 2a-d). SNPs per chromosomes ranged from 2867 (chr8) to 4662 (chr3) and SNP density per chromosome (SNPs/Mbp) ranged from 54.3 (chr4) to 66.2 (chr9) (Fig. 2e).

Of the 31,811 polymorphic markers, $7372(23.2 \%)$ were shared among all supopulations (Fig. 2d). By subpopulation, 


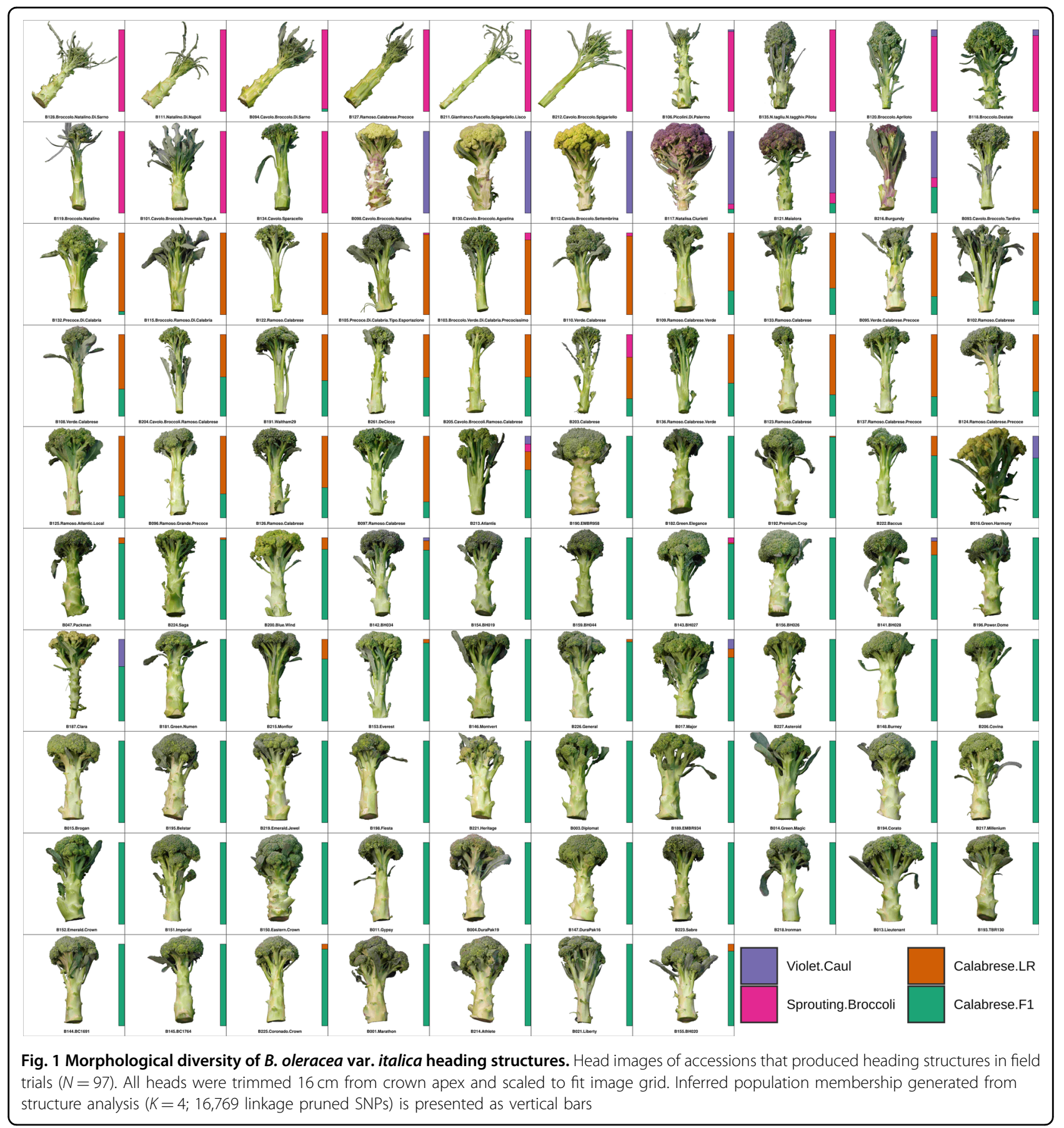

Calabrese hybrid accessions contained the fewest unique polymorphic alleles $\left(8.3\right.$ accession $\left.^{-1}\right)$ and sprouting broccoli types contained the most $\left(39.1\right.$ accession $\left.^{-1}\right)$. When comparing the polymorphic markers between the inferred Calabrese hybrid and Calabrese landrace subpopulations, $49.7 \%$ were common to both (Calabrese hybrid $\cup$ Calabrese landrace $=26,259$; Calabrese hybrid $\cap$ Calabrese landrace $=$ 13,052; Calabrese landrace - Calabrese hybrid $=9,490$ ), and Calabrese landraces contained 4.8 -fold greater unique alleles per accession compared to Calabrese hybrid accessions.

When comparing the inferred Calabrese hybrid subpopulation against all other taxa, site missingness was not significantly higher (Fig. 2a), although heterozygousity was reduced in the same comparison (Fig. 2b; $p<0.01$ ). We observed a right skew and an increase in minor allele variants in Calabrese hybrids compared to other italica types $(p<0.01)$. For all accessions pooled across all 


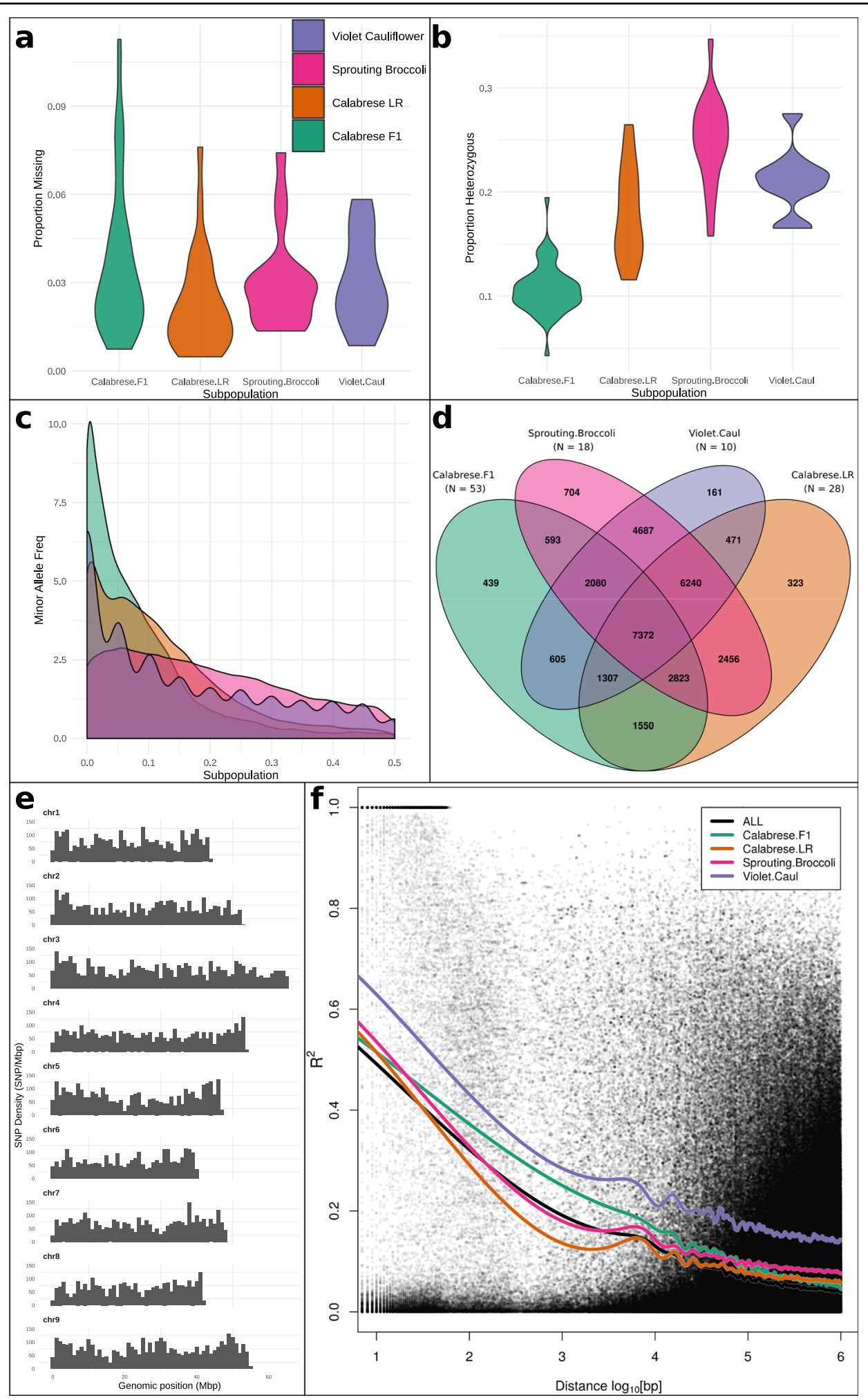

Fig. 2 GBS summary statistics for $\mathbf{3 1 , 8 1 1}$ high-quality SNP markers. a Proportion of site missingness across all markers by subpopulation. b Proportion of site heterozygousity across all markers by subpopulation. c Pooled minor (second most common) allele frequency by subpopulation. d Unique polymorphic SNPs by subpopulation. e SNP density by chromosome (chr1-chr9) and genomic position in 1 Mbp windows ( $x$-axis). $\mathbf{f}$ Linkage disequilibrium decay plotted as pairwise $r^{2}$ against $\log _{10}(\mathrm{bp})$ with subpopulation data fitted with cubic smoothing spline $($ spar $=0.5)$ 
markers, linkage disequilibrium decayed rapidly to background levels $\left(r^{2}<0.2\right)$ by $0.82 \mathrm{kbp}$ (Fig. 2f). Linkage decay to background levels was substantially different by subpopulation, decaying fastest in the Calabrese landraces $(0.30 \mathrm{kbp})$, followed by sprouting broccoli $(0.63 \mathrm{kbp})$, Calabrese hybrids $(4.12 \mathrm{kbp})$, and violet cauliflower (52.1 kbp).

Of the SNP variants identified between markers and the reference genome, 4,523 were predicted to result in missense, nonsense (129), and silent $(5,195)$ amino acid changes [Supplementary Data 1 (variants.ods)]. In several agricultural crops, modern accessions exhibit AT-bias across polymorphic sites compared to their respective landraces ${ }^{62}$, and this finding was confirmed when comparing modern Calabrese broccoli hybrids against the less improved italica germplasm. Mean genome-wide [AT] base composition was highest in the Calabrese hybrid subpopulation and different between subpopulations $(p<0.01$; sprouting broccoli $=0.312<$ violet cauliflower $=$ $0.333<$ Calabrese landraces $=0.344<$ Calabrese hybrids $=$ $0.373)$.

\section{Diversity analysis \\ Principal components}

The genetic diversity, phylogeny, and population structure of 109 distinct B. oleracea accessions was evaluated using 31,811 genome-wide markers. Principal component analysis (PCA) using these markers effectively resolved the 109 accessions into four subpopulations and the first three axes explained a cumulative $74.6 \%$ of model variation (Fig. $3 \mathrm{a}-\mathrm{c}$ ). The Calabrese accessions were clearly resolved from other accessions, and PCA axis 3 formed a gradient between the Calabrese subpopulations, with early modern open-pollinated Calabrese accessions B261.DeCicco and B191.Waltham29 located between the landrace and hybrid subpopulations, and recently released $\mathrm{F}_{1}$ hybrids were located at the extremity. The tropical cauliflowers B187.Clara and B016.Green.Harmony and the violet cauliflower $F_{1}$ hybrid B216.Burgundy was located between the Calabrese hybrid and violet cauliflower groups, consistent with an admixed breeding pedigree. Calabrese landraces were collected along the entire Italic Peninsula, whereas the sprouting broccoli and violet cauliflower accessions were collected nearly exclusively in the Southern Italic Peninsula and Sicily and PC axis 1 versus collection latitude exhibited the strongest association with collection location $\left(R^{2}=0.45\right)$ (Fig. 3d). $\mathrm{PC}$ axis 2 was most correlated with cultivar release year $\left(R^{2}=0.50\right)$. SNP coefficients explaining PCA variance ranged from -0.027 to +0.050 , and the top $1 \% \mathrm{PC}$ coefficients by absolute value were retained for further analysis. Of these high-loading SNPs, 762 (41.6\%) were located within gene intervals, and 13 (Bo1g021960, Bo1g039650, Bo1g051570, Bo2g018320, Bo2g041560,
Bo3g001090, Bo3g080160, Bo3g094030, Bo4g045930, Bo5g010600, Bo5g150300, Bo6g080130, Bo7g088960) were marked as high impact variants [Supplementary Data 1 (variants.ods)].

\section{Phylogeny, identify-by-state, and structure}

Phylogenetic analysis identified four main subpopulations (Fig. 4a, Supplementary Fig. 2). All Calabrese accessions formed a monophyletic clade, and the Calabrese hybrids formed a monophyletic clade within the Calabrese clade. The violet cauliflower accessions and the sprouting broccoli subpopulations formed monophyletic and a paraphyletic clades, respectively.

Identity by state analysis was used to construct a similarity matrix using all lines (Fig. $4 \mathrm{~b}$ ). Mean probability of identity by state $\left(\mathrm{P}_{\mathrm{IBS}}\right)$ was 0.80 across all lines and pairwise $\mathrm{P}_{\mathrm{IBS}}$ ranged from 0.70 (B212.Cavolo.Broccolo.Spigariello versus B100.Cavolo.Broccolo.Marzullo) to 0.95 (B193.TBR130 versus B013.Lieutenant). When comparing individual accessions to all other accessions, the most distinct and similar accessions were B106.Picolini.Di. Palermo (mean $P_{\mathrm{IBS}}=0.73$ ) and B001.Marathon (mean $\left.P_{\mathrm{IBS}}=0.84\right)$. Two groups of Calabrese landraces were very similar $\left(P_{\mathrm{IBS}}>0.90\right)$ : the nonheading accessions (B107. Ramoso.Calabria.Mezzo.Precoce, B129.Ramoso.Calabria. Tardivo, and B131.Broccolo.Verde.Di.Calabria.Tardivo) and four small-headed Calabrese accessions (B095.Verde. Calabrese.Precoce, B102.Ramoso.Calabrese, B108.Verde. Calabrese, B109.Ramoso.Calabrese.Verde, and B133. Ramoso.Calabrese). Several early commercial landraces (B191.Waltham29, B204.Cavolo.Broccoli.Ramoso.Calabrese, B205.Cavolo.Broccoli.Ramoso.Calabrese, and B261. DeCicco) and accessions from the USDA-USVL breeding program (B143.BH027, B154.BH019, B156.BH026, and B159.BH044) appeared highly related ( $\left.P_{\mathrm{IBS}}>0.90\right)$.

Several interesting patterns of relatedness were observed in population structure analysis (Fig. 4c). Members of the green population were exclusively Calabrese $F_{1}$ hybrids (mean membership $=95.1 \%$; range $=58.9-100.0 \%$ ). Majority members of the orange population were exclusively identified as Calabrese landraces $(N=28$; mean membership $=77.6 \%$; range $=50.6-100.0 \%$ ). The pink majority subpopulation was comprised entirely of accessions collected from the Southern Italic Peninsula and Sicily $\quad(N=18 ; \quad$ mean $=91.4 \% ; \quad$ range $=52.0-100.0 \%)$. Majority members of the purple subpopulation $(N=10$; mean membership $=91.7 \%$; range $=56.7-100 \%$ ) was comprised entirely of purple cauliflower types that were characterized by an intermediate italica and botrytis phenotype, a purple to off-white heading inflorescence, intermediate meristem arrest stage, long connected petiole wings, and little to no lateral shoot formation.

Calabrese landraces were partially admixed with the Calabrese hybrid subpopulation (mean $=20.9 \%$; 


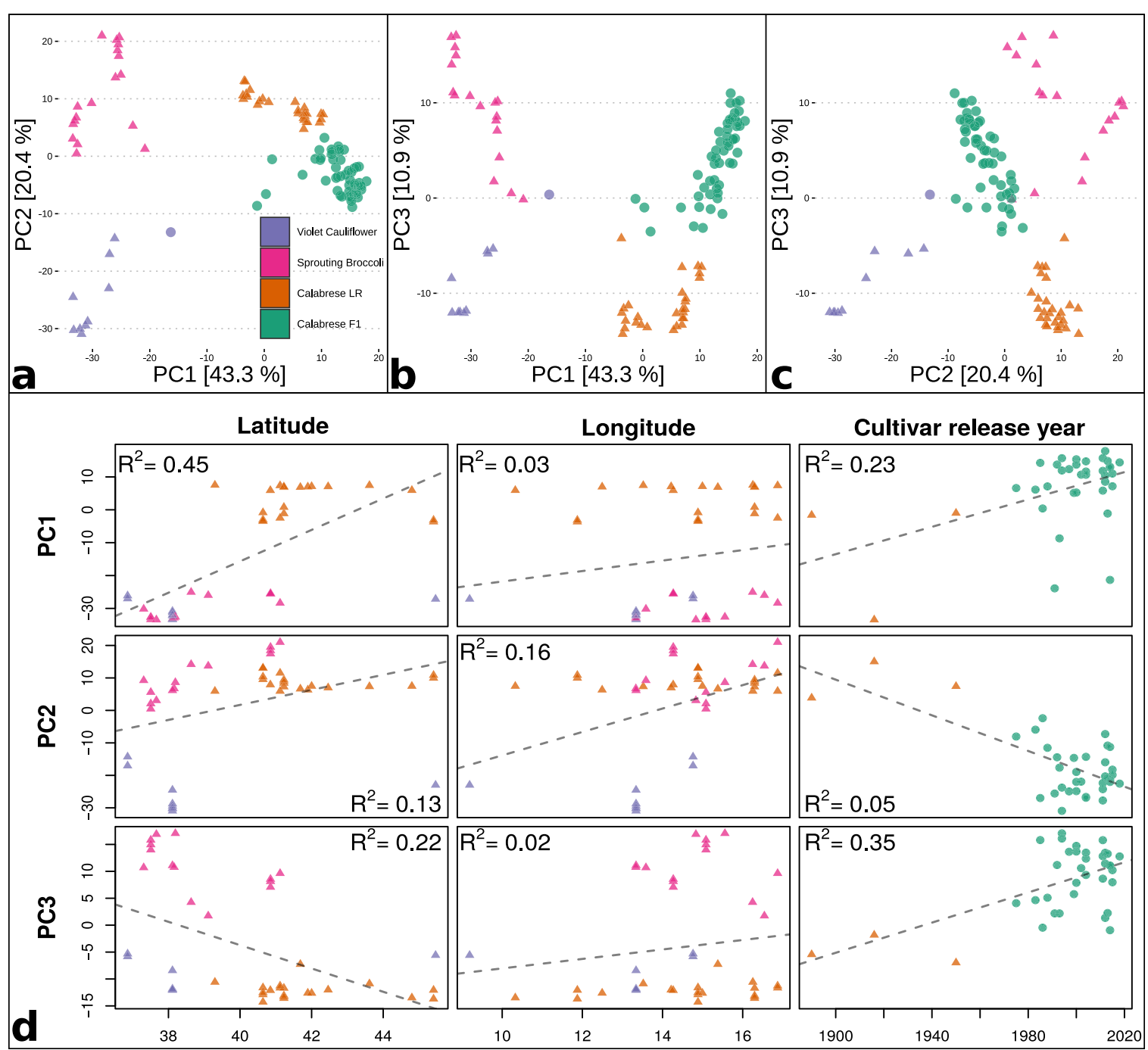

Fig. 3 Patterns of population structure by principal component analysis of accessions using 31,811 SNP markers with colors assigned by inferred subpopulation (green $=$ Calabrese hybrids, orange $=$ Calabrese landraces, pink $=$ sprouting broccoli, purple $=$ violet cauliflower, landrace $=$ triangles, hybrid $=$ circles). a-c Pairwise comparisons between multidimensional scaling axes of PC axes 1-3 with percent variance explained printed along axes. $\mathbf{d}$ Nonstratified linear model comparing PC axes 1-3 [rows] against accessions with geographical provenances [latitude $\left({ }^{\circ} \mathrm{N}\right)$, longitude $\left({ }^{\circ} \mathrm{W}\right)$, and cultivar release year; columns] with model $R^{2}$ printed in subplots

range $=0.0-48.1 \%$, and some Calabrese hybrids were partially admixed with the Calabrese landrace subpopulation (mean membership $=3.0 \%$; range $=$ 0.0-24.1\%), including the accessions B215.Monflor (24.1\%), 'Packman' related B222.Baccus (23.9\%), and the alboglabra x italica hybrid B213.Atlantis (22.2\%). Members of the sprouting broccoli and violet cauliflower subpopulations shared very little membership with the Calabrese hybrid (mean $=1.2 \%$ and $5.2 \%$ ) or Calabrese landrace subpopulations ( mean $=3.9 \%$ and $0.0 \%$ ).

The sprouting broccoli and violet cauliflower subpopulations have experienced very little admixutre with each other or Calabrese types. The sprouting broccoli (pink) subpopulation component was almost never identified within other subpopulations (Calabrese hybrid $=0.0 \%$, Calabrese landrace $=1.5 \%$, violet cauliflower $=0.0 \%$ ), and the violet cauliflower subpopulation (purple) was uncommon in members of other subpopulations (Calabrese hybrid $=$ $1.7 \%$, Calabrese landrace $=0.0 \%$, sprouting broccoli $=3.4 \%$ ).

However, several italica/botrytis phenotypic intermediates were confirmed in structure analysis: the accession B216.Burgundy is a $\mathrm{F}_{1}$ hybrid with an italica $x$ botrytis pedigree $(56.7 \%$ purple and $31.6 \%$ green). Two Calabrese hybrid majority members are commercial tropical cauliflower hybrids with temperature-sensitive heading structure and contained partial admixture within the purple subpopulation (B016.Green.Harmony $=26.8 \%$, B187. Clara $=33.2 \%$ ). 


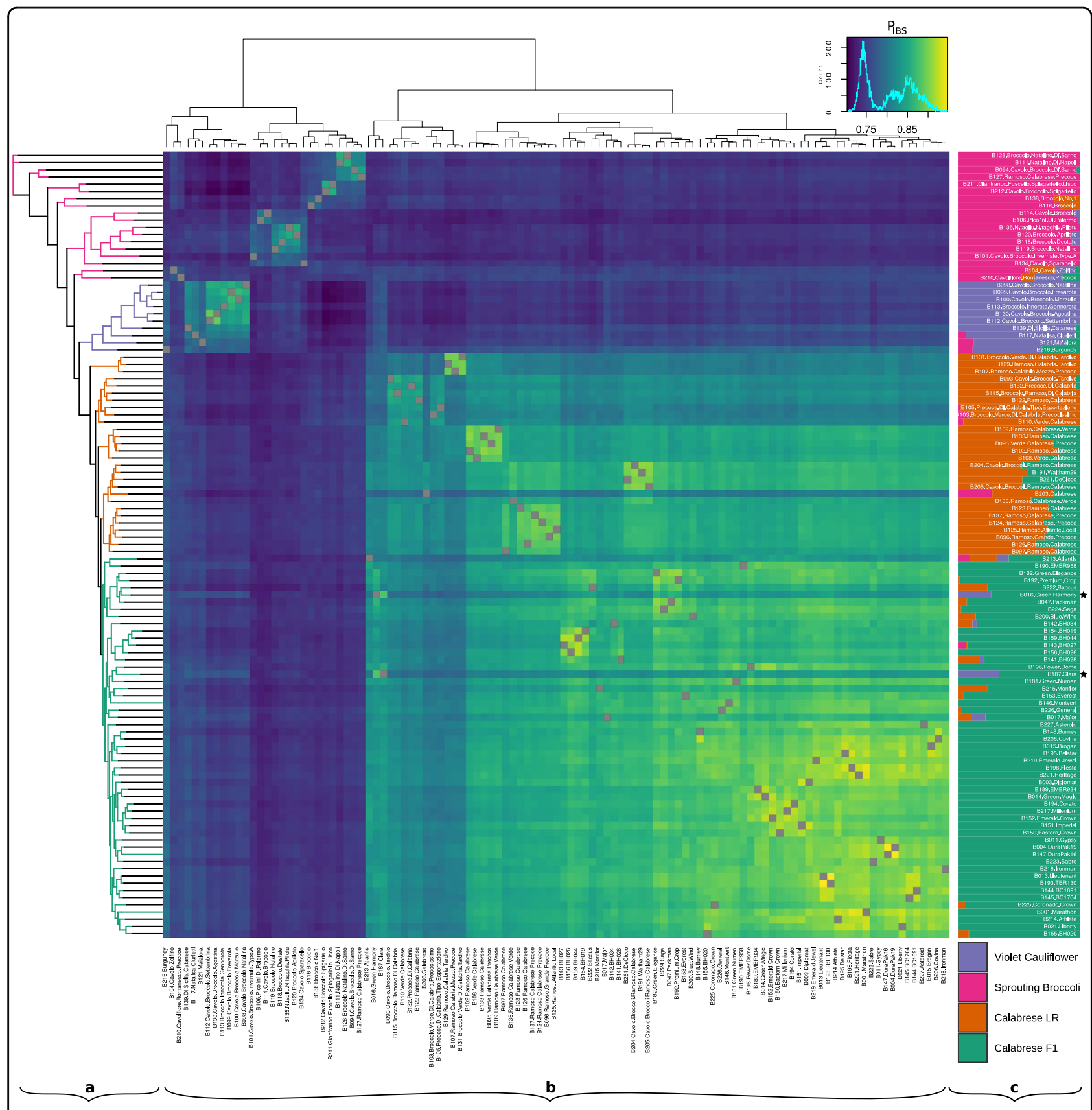

Fig. 4 Three analyses of relatedness of all evaluated accessions $(\boldsymbol{N}=\mathbf{1 0 9})$. a Maximum-likelihood based phylogenetic inference using 31,811 SNPs (GTR + GAMMA substitution model, $N=100$ ), indicating four subpopulations (legend bottom right; violet cauliflower $=$ purple, sprouting broccoli = pink, Calabrese broccoli landraces = orange, Calabrese broccoli hybrids = green). b Similarity matrix of all accessions calculated as probability of identity by state, averaged over all non-missing loci (legend top; range $0.70-0.95$ ), indicating genetic similarities between accessions and inferred subpopulations. Similarity matrix rows are sorted by RAXML phylogenetic inference order and columns are sorted by genetic similarity between genotypes. c Proportion of subpopulation membership of accessions within via FastStructure analysis using 16,341 linkage pruned SNPS $\left(K=4\right.$; legend bottom right). Rows are sorted by phylogenetic inference, and tropical cauliflower accessions indicated by $\left(^{*}\right)$

\section{$\mathrm{F}_{\text {st }}$, reduction in diversity, selective sweeps, and runs of homozygosity}

Several notable genomic patterns were revealed when comparing the Calabrese hybrid subpopulation against all other accessions (Fig. 5). Fixation index $\left(\mathrm{F}_{\mathrm{st}}\right)$ is a measure of structure related population differences. Genome-wide scans comparing Calabrese hybrids against all other accessions identified 24 genome-wide $F_{\text {st }}$ enriched regions in modern Calabrese hybrid germplasm [Supplementary Data 2,.(genomic-regions.ods)]. Pooled weighted $\mathrm{F}_{\mathrm{st}}$ between 


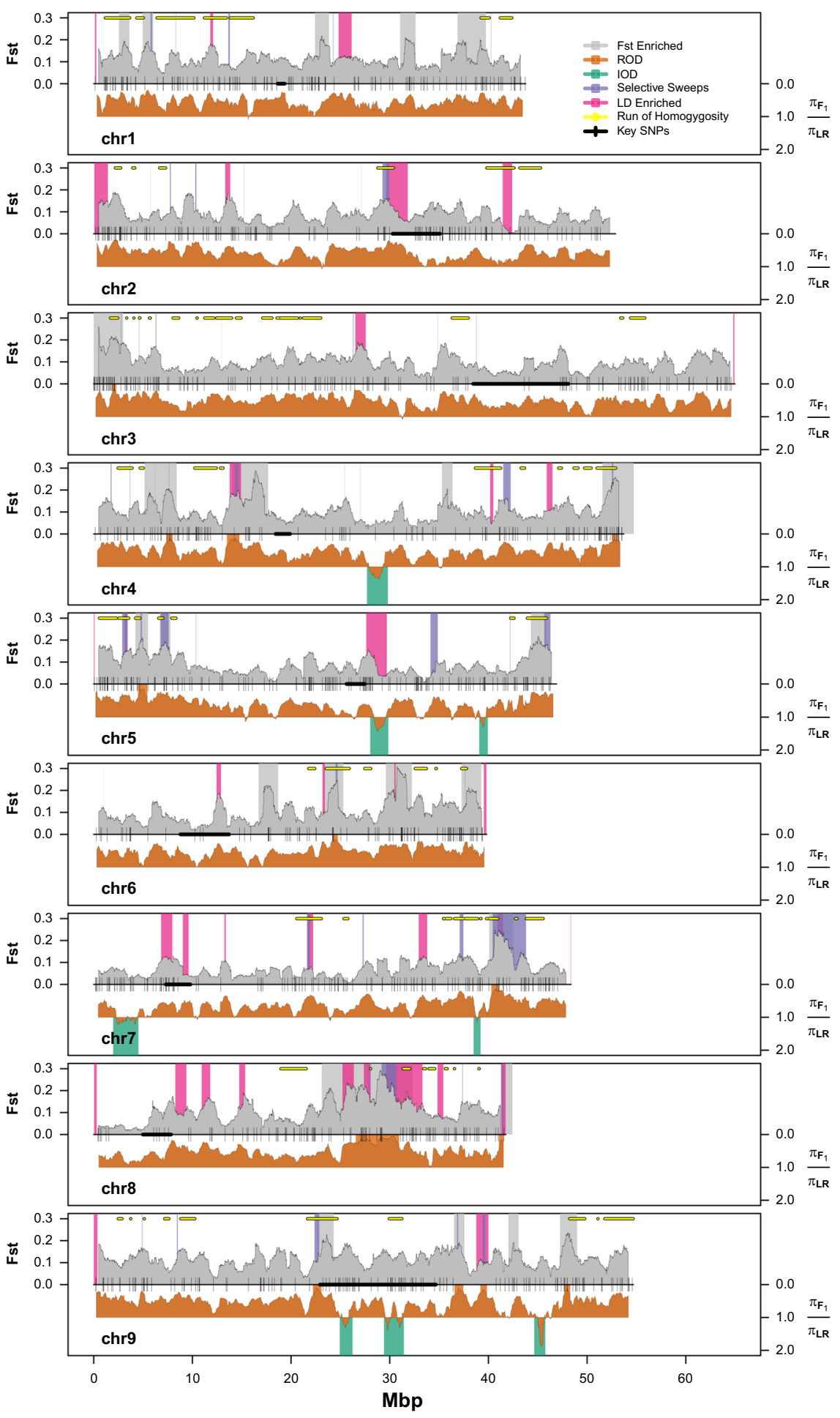

Fig. 5 Genome-wide scans of selection and domestication footprints when comparing Calabrese hybrids with italica landraces using 31,811 SNPs markers by chromosome (chr1-chr9) and physical position (Mbp; $\boldsymbol{x}$-axis). Fixation index [ $\mathrm{F}_{\text {sti }}$ gray trace; left $y$-axis] and ratio of nucleotide diversity [orange trace; right $y$-axis] comparing Calabrese hybrids against all other accessions, with enriched $F_{\text {st }}$ regions printed in gray boxes, with top reduced $(\mathrm{ROD}=$ orange) and enriched $(\mathrm{IOD}=\mathrm{green})$ nucleotide diversity regions printed as boxes. Selective sweeps (purple) and linkage disequilibrium enriched (pink) regions are printed as boxes. Pooled runs of homozygosity identified within the Calabrese hybrids are printed as yellow bars. Key PCA loading SNPs and centromeric regions derived from half-tetrad analysis ${ }^{6}$ are printed as black ticks and bold centerlines, respectively 
subpopulations were moderately low between Calabrese landraces and hybrids $\left(F_{\mathrm{st}}=0.09\right)$, and very high between Calabrese hybrids and violet cauliflower $\left(F_{\mathrm{st}}=0.33\right)$ and sprouting broccoli $\left(F_{\mathrm{st}}=0.26\right)$. Calabrese landraces were less differentiated between the violet cauliflower $\left(F_{\mathrm{st}}=0.25\right)$ and sprouting broccoli $\left(F_{\mathrm{st}}=0.16\right)$ subpopulations. The sprouting broccoli and violet cauliflower populations were moderately differentiated $\left(F_{\mathrm{st}}=0.14\right)$. Fifteen regions (Fig. 5 [gray]) with elevated $\mathrm{F}_{\text {st }}$ were identified and contained 4208 genes. Eight of these elevated $\mathrm{F}_{\text {st }}$ region genes were identified as high impact variants (Bolg078320, Bo3g001090, Bo4g196000, Bo5g014760, Bo6g079470, Bo6g080130, Bo6g099010, Bo8g088460) [Supplementary Data 3 (genomic-regions.ods)].

Selection driven sweeps can result in reduced genetic diversity in improved germplasm compared to undomesticated or landraces germplasm. Overall, Calabrese hybrids experienced considerable genome-wide reduction in nucleotide diversity when compared to all other accessions (ROD $=0.55$; Fig. 5 [orange]). In this comparison, 13 reduced and 8 enriched nucleotide diversity genomic regions were identified (Fig. 5) spanning on average $1.0 \mathrm{Mbp}$ (range $=0.4-4.4 \mathrm{Mbp}$ ) and 1.57 $\mathrm{Mbp}$ (range $=0.7-2.6 \mathrm{Mbp})$, respectively. Some of the 13 regions were remarkably reduced in nucleotide diversity; for example, the chr8:29.8-30.6 Mbp region, ROD was reduced to 0.05 . Of the 8 enriched diversity regions in the Calabrese hybrid subpopulation, three were identified on chromosome 9. The most notable enriched diversity region $(\mathrm{ROD}=1.87)$ was located near the end of chromosome 9 (chr9:44.6-45.8 Mb). Of the 1906 and 1174 genes contained in the reduced and enriched intervals, three reduced (Bo5g014760, Bo6g080130, Bo8g088460) and two enriched (Bo5g126850 and Bo7g009560) SNPs were designated as high impact variants.

Analysis of the Calabrese hybrid subpopulation identified 53 high-likelihood selective-sweep intervals spanning from 0.1-3.4 Mbp (likelihood = 9.3 to 17.3; Fig. 5 [purple]). These intervals contained 1861 genes, and six were classified as high-impact variants (Bo1g017170, Bo5g113490, Bo5g113510, Bo5g148470, Bo5g150300, Bo8g088460).

Runs of homozygousity are predictors of whole-genome inbreeding, and longer runs are evidence of more recent selective pressure ${ }^{63}$. When scanning all accessions, we identified $88(>1 \mathrm{Mbp}$ ) runs of homozygosity (Fig. 5 [yellow]). These regions were nearly exclusively comprised of Calabrese hybrid accessions and $11.4 \%$ of these regions included 10 or more Calabrese hybrid accessions.

We detected four unusually feature-rich genomic regions (chr4:14.3-14.6; chr7:40.9-41.1; chr8:27.9-30.7, and chr8:41.4-41.5) that exhibited evidence of selective sweeps, elevated $F_{s}$, high LD, and high ROD. Of the 24 elevated $\mathrm{F}_{\text {st }}$ regions, there were 18 instances of a selectivesweep (75.0\%) and 16 instances (66.7\%) of a run of homozygousity collocating with a given $F_{\text {st }}$ region. Selective sweeps were never identified within enriched diversity regions, but were identified in 12 of the 13 reduction in diversity regions. The only reduced diversity region without a selective sweep was chr9:47.7-48.3 Mbp, adjacent to the strongest enriched diversity region (chr9:44.6-45.8 Mb).

\section{Phenotyping}

To capture the diversity contained within italica germplasm, seed from 54 commercial $\mathrm{F}_{1}$ hybrid broccoli and 55 landrace/open-pollinated accessions was collected from as many breeding programs and distribution channels as possible: Asgrow (2), Bejo (5), Emerald (2), Enza-Zaden (1), Evergrow (2), the USDA Agricultural Research Service (7), Harris-Moran (1), Johnny's Seed (4), Known-You Seed Company (2), Northeast Seed (1), Peto Seed (2), Sakata (10), Seminis/Vaden Bosch/Royal Sluis (8), Syngenta (4), Tainong (2), and Takii (1) (Supplementary Table 1, Fig. 1). Of the 47 landrace accessions collected in Italy, 43 contained geographic provenances (range $36.86-45.46{ }^{\circ} \mathrm{N}$ and 9.19-16.87 ${ }^{\circ}$ E; Fig. 6a).

In total, 24 horticultural quality traits within four trait categories were evaluated within two trial environments (Supplementary Table 2, Fig. 6b-d, Supplementary Data 3 [pheno.csv]) to compare morphological changes of modern Calabrese broccoli hybrids against the Calabrese landraces and the larger pool of italica diversity. To identify trends in improvement, traits were regressed against the year of cultivar release.

\section{Architectural}

Plant size at maturity is an important yield characteristic, with more compact plants allowing higher density plantings. The architectural traits plant height and width ranged from 22.0 (B189) to 86.4 (B213) $\mathrm{cm}$ tall and 38.9 $(\mathrm{B} 190)$ to 97.8 (B111) $\mathrm{cm}$ wide and were different between inferred subpopulations $(p<0.01)$. The Calabrese hybrid subpopulation was the shortest and narrowest $(41.0 \times 68.9 \mathrm{~cm})$. Plant height and width was not correlated with cultivar release year. Higher head extension above leaf rosette enables efficient harvest and was different between subpopulations $(p<0.01)$ and negatively correlated with heading quality $\left(r_{\mathrm{s}}=-0.32\right)$, harvest index $\left(r_{\mathrm{s}}=-0.65\right)$, cultivar release year $(p=$ $0.02)$. Calabrese landrace accessions exhibited greater head extension (3.83) when compared to Calabrese hybrid accessions $(3.02 ; p<0.01)$.

A single central head is a primary goal in broccoli breeding programs as lateral shoots increase harvest difficulty. The number of lateral shoots per plant ranged from 0.0 (ten accessions) to 42 (B211) and was negatively correlated overall quality $\left(\mathrm{r}_{\mathrm{s}}=-0.57\right)$ and cultivar release 


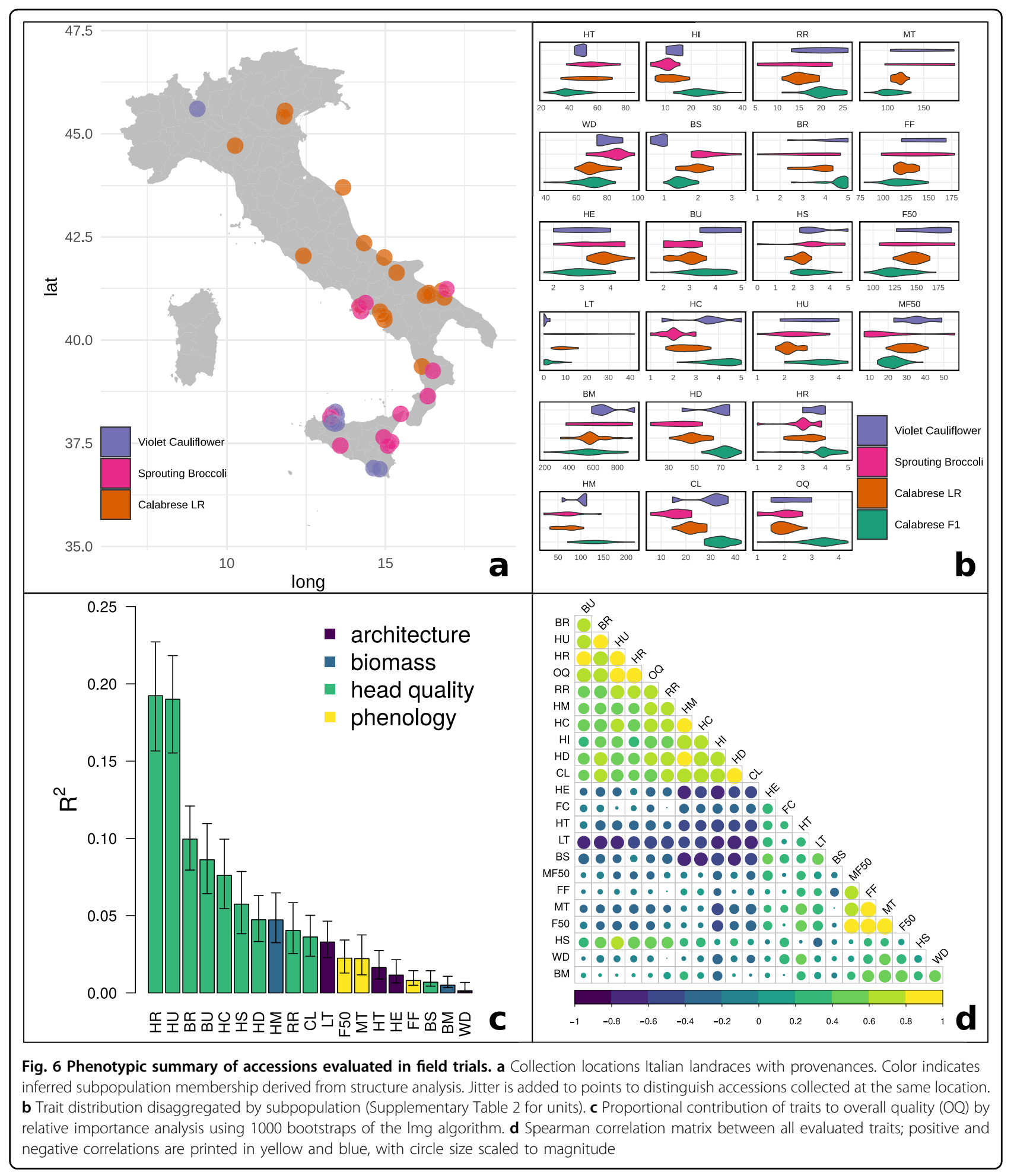

year $(p<0.01)$. The Calabrese hybrid subpopulation exhibited fewer lateral shoots (3.0) than Calabrese landraces (9.4) and sprouting broccoli (16.8), but more than violet cauliflower (0.6).

\section{Biomass}

While above-ground plant biomass has not increased over time, head mass and harvest index has, consistent with the breeding objective of enabling denser plantings 
by producing larger and heavy heads from compact plants. All biomass traits evaluated were different between inferred subpopulation $(p<0.01)$. Above-ground biomass ranged from 193.1 (B187) to $941.9 \mathrm{~g}$ (B098) and head mass ranged from 18.8 (B212) to 218.4 g (B190). Calabrese hybrid heads were heavier (128.8 g) than Calabrese landraces $(68.1 \mathrm{~g})$, sprouting broccoli $(66.7 \mathrm{~g})$, and violet cauliflower $(98.3 \mathrm{~g})$ heads. Head mass was positively associated with cultivar release year $(p<0.01)$ with a slope of $+0.89 \mathrm{~g} \mathrm{year}^{-1}$. Harvest index ranged from $4.2 \%$ to (B211) to 39.6\% (B187).

Harvest index was positively correlated cultivar release year $(p<0.01)$ and mean Calabrese hybrid harvest index (23.2\%) was nearly double that of Calabrese landrace landraces (12.0\%), indicative of strong selection pressure during improvement.

\section{Head quality}

Improved head quality is a primary target improvement and is required for high quality, regionally adapted cultivars. Average bead size at harvest ranged from to $0.6 \mathrm{~mm}$ (B130) to $3.28 \mathrm{~mm}$ (B094) and was negatively associated with cultivar release year $(p<0.01)$. The inferred subpopulations differed in bead size, and modern broccoli hybrids (Calabrese hybrid $=1.50 \mathrm{~mm}$ ) have smaller beads than broccoli landraces (Calabrese landrace $=1.92 \mathrm{~mm}$ ). The violet cauliflower subpopulation exhibited the highest flower bud uniformity (4.2) compared to the Calabrese hybrid (3.6), Calabrese landrace (2.9), and sprouting broccoli (2.7) subpopulations, and bud uniformity was positively correlated with cultivar release year $(p=0.023)$.

The head mass characteristics: head compactness, head diameter, average cluster width, and first rank branching were all different between subpopulation $(p<0.01)$. Head compactness was positively associated with cultivar release year $(p<0.01)$, ranging from 2.0 (sprouting broccoli) to 4.1 (Calabrese hybrids). Head diameter per accession ranged from $16.1 \mathrm{~mm}$ (B211) to $86.0 \mathrm{~mm}$ (B004) and was positively associated with cultivar year release $(p<0.01)$. Between subpopulations, head diameter ranged from $37.0 \mathrm{~mm}$ (sprouting broccoli) to $72.6 \mathrm{~mm}$ (Calabrese hybrids) and Calabrese hybrid head diameter was $23.6 \mathrm{~mm}$ greater compared to the Calabrese landrace accessions. Average cluster width and first rank branching were correlated with cultivar release year $(p<0.01)$. Per subpopulation, cluster width and first rank branching ranged from 15.9 (sprouting broccoli) to $34.1 \mathrm{~mm}$ (Calabrese hybrid) for cluster width and 15.2 (sprouting broccoli) to $20.6 \mathrm{~mm}$ (Calabrese hybrid) for first rank branching.

The presence of cauline leaves bisecting the heading structure at maturity is a common quality flaw in heat sensitive broccoli cultivars. Bracting suppression ranged from complete (5.0; B004) to none (1.0; B101) but was not significantly associated with cultivar release year. Bracting suppression was different between subpopulations $(p<$ 0.01 ) and stronger in Calabrese hybrid (4.5) compared to Calabrese landrace accessions (3.6).

A convex and uniform crown shape is required for high quality broccoli production and helps to shed water during the pre-harvest interval. Head morphology traits were different between inferred subpopulations $(p<0.01)$, but were not strongly associated with cultivar release year. Between subpopulations, head shape ranged from 2.4 (Calabrese landrace) to 3.3 (violet cauliflower), and head uniformity ranged from 2.2 (Calabrese landrace) to 3.3 (Calabrese hybrid).

Breeding efforts to expand the adapted range of broccoli have focused on decreasing the minimum chilling hour requirements for normal heading and increasing overall heat tolerance. Sensitive hybrids experience flaws at the meristem arrest and proliferation stages, resulting in a failure to produce heads, irregular flower bud sizes, and other defects. Heat tolerance ranged from none (1.0; B101) to very high (5.0; B141) and was strongly correlated with overall quality $\left(R^{2}=0.78\right)$, but not with cultivar release year. On average, the Calabrese hybrid subpopulation (3.8) exhibited greater heat tolerance $(p<0.01)$ compared to the Calabrese landrace subpopulation (3.1).

Overall quality, an aggregate measurement of horticultural quality within a given environment has been used to select optimal hybrids in breeding trials ${ }^{52}$. Here, overall quality ranged from very low $(1.0 ; \mathrm{B} 101)$ to very high (4.33; B206) and was associated with cultivar release year $(p=0.02)$. Per inferred subpopulation, overall quality was different $(p<0.01)$, ranging from 1.9 (sprouting broccoli) to 3.2 (Calabrese hybrid). Calabrese hybrid accessions performed +1.2 points better than Calabrese landrace accessions $(p<0.01)$. Relative importance analysis indicated that variation in heat response $(19.0 \%)$ and head uniformity (19.0\%) explained the greatest amount of variation in overall heading quality (OQ) and the remaining modeled traits cumulatively explained less than $16.4 \%$ of variation in overall quality (Fig. 6c).

Flower color was predominately yellow, although six sprouting broccoli accessions produced white flowers (B118, B119, B120, B135, B211, and B212).

\section{Phenology}

All of the phenology traits were strongly correlated between each other, different between subpopulations, and not correlated with cultivar release year. Days to from sowing to head maturity ranged from 67.5 (B187) to $193 \mathrm{~d}$ (B094 and B128), with 16 accessions failing to consistently head. Calabrese hybrid types reached head maturity the fastest $(103.8 \mathrm{~d})$ and the sprouting broccoli types the slowest (152.7 d). Days to first flowering ranged from 75.3 (B016) to $178.5 \mathrm{~d}$ (B127) and 21 lines did not achieve any 
flowering. Calabrese hybrid accessions flowered the earliest $(119.4 \mathrm{~d})$ and the violet cauliflower the latest (147.2 d). Days to 50\% flowering ranged from 89.7 (B016) to 194 (B119) and 22 lines did not achieve 50\% flowering. Days from head maturity to $50 \%$ flowering ranged from 7.0 (B127) to 56.0 (B119), although B119 and several other landrace accessions exhibited highly heterogeneous within-plot heading and flowering that likely inflated this value. By subpopulation, days from head maturity to $50 \%$ flowering varied from 21.5 (sprouting broccoli) to $35.7 \mathrm{~d}$ (violet cauliflower).

\section{Discussion}

To our knowledge, this study provides the most comprehensive investigation of the morphological and genomic diversity of $B$. oleracea var. italica germplasm. Using genotype-by-sequencing, we generated 31,811 highquality SNP markers with a mean density of 65.2 SNPs/ Mbp which is suitable for subsequent GWA studies given genome-wide linkage disequilibrium. These markers were used to infer four subpopulations by evaluating principal components, phylogeny, identity-by-state, population structure. We then evaluated critical genomic regions and targets of selection in modern $F_{1}$ Calabrese broccoli by evaluating genome-wide population differentiation, reduced or enriched nucleotide diversity, selectivesweeps, and runs of homozygosity (Figs. 2-4). These 109 unique landrace and $F_{1}$ accessions were phenotyped for 24 horticultural traits within two growth environments (Figs. 1, 6b, c).

Modern $\mathrm{F}_{1}$ hybrid Calabrese broccoli was delineated as a monophyletic clade within a larger monophyletic clade of Calabrese broccoli and was clearly differentiated by principal component and structure analysis. We observed lower heterozygosity in the Calabrese $F_{1}$ hybrids compared to the other inferred subpopulations, a somewhat unexpected result given that $F_{1}$ hybrids crossing schemes typically aim to increase heterozygosity. This result is the most consistent with repeated crossing within a pool of highly homogeneous parental germplasm used to generate Calabrese $F_{1}$ hybrids. Our results are consistent with a reduction in genetic diversity that occurred during breeding efforts in the last century, eroding the genetic base of Calabrese broccoli germplasm. Individually and as a group, hybrid Calabrese broccoli accessions were more homozygous, had greater within-group similarity, and exhibited considerably reduced allelic diversity (Figs. 2d and $4 \mathrm{a}-\mathrm{c}$ ). In addition, there was a positive [AT] nucleotide composition bias in hybrid Calabrese accessions, concordant with results previously identified in elite maize and soybean cultivars ${ }^{62}$.

We expected to identify selective sweeps in the vicinity of genes that regulate horticultural quality traits under selection during domestication and further crop improvement. Several key genomic regions bear strong signals of domestication and selection, and warrant further investigation. This terminal chromosome 9 region has been shown to harbor homologs of flowering and chilling requirement genes TLF2, COL1, CO, and $F L C^{32,64-67}$ and has been linked to temperaturedependent time to curd initiation in a double-haploid cauliflower $^{68}$, heat-tolerance in a double-haploid italica mapping population ${ }^{69}$, and a head forming and later flowering time phenotype in a double-haploid alboglabra $\mathrm{x}$ italica mapping population ${ }^{27}$. We identified a strong $\mathrm{F}_{\mathrm{st}}$ region (chr9:47.4-48.9) that contained 236 A. thaliana homologs including $\mathrm{CO}$ (Bo9g163730) and COL1 (Bo9g163720). Interestingly, this region was flanked by the strongest genome-wide increase in diversity region (chr9:44.6-45.8 Mb). A region of increased diversity flanking a decreased diversity region would be expected given the casual gene in the decreased diversity region was a repeated target for backcrossing. These results support previous work that the chromosome $950 \mathrm{Mb}$ region is a likely target for domestication and improvement in Calabrese broccoli. In Calabrese broccoli hybrids, the largest and strongest region of population differentiation was a 7.5 Mbp region (chr8:23.4-30.9) that collocated with a region of $95 \%$ reduced nucleotide diversity (chr8:26.6-31.0 Mb), three linkage disequilibrium enriched blocks, three selective sweeps intervals, and was flanked by several runs of homozygosity. This region contained 26 of the top 1\% SNPs associated with variation in PCA population differentiation.

Calabrese hybrids exhibited superior horticultural quality characteristics compared to other italica types, including Calabrese landraces. Unlike many $F_{1}$ hybrid crops when compared to their respective landraces, Calabrese broccoli hybrids did not necessarily produce larger plants, rather producing larger, heavier, more compact and uniform heads, which comprised a larger fraction of total above-ground biomass. When comparing head mass by year of cultivar release, on average, breeding efforts in the last 130 years have increased total head mass by $\sim 0.9 \mathrm{~g}$ per year. Modern hybrid Calabrese broccoli heads exhibited greater bracting suppression and more uniform flower buds when compared to Calabrese broccoli landraces. The linkage of lower head extension with other quality traits could result from hitchhiking or as a pleiotropic effect resulting from selection for stronger meristem arrest.

Although no collected Calabrese broccoli landraces were collected in Sicily, they were collected throughout the Italic Peninsula, and not exclusively in Calabria (Fig. 6a). These accessions are clearly morphologically recognizable as Calabrese broccoli, although they exhibited slower head maturity and flowering and inferior heading qualities compared to modern hybrid Calabrese broccoli. Two 
historically important commercial open-pollinated accessions, B261.DeCicco (1890) and B191.Waltham29 (1950), were partially admixed in structure analysis with modern hybrid Calabrese broccoli, and this relationship was confirmed by principal component analysis. These historical commercial types share close genetic similarity to other Calabrese landrace accessions such as B204. Cavolo.Broccoli.Ramoso.Calabrese and B205.Cavolo. Broccoli.Ramoso.Calabrese. These accessions are, or are closely related to, the founding germplasm exploited during the initial breeding of modern Calabrese hybrids.

Sprouting broccoli accessions were collected either in Sicily or the southern Italian Peninsula below $41{ }^{\circ} \mathrm{N}$ and exhibited fewer signals of selection and domestication compared to other broccoli types. The sprouting broccoli morphotype was characterized by large plants with many lateral shoots, long but narrow leaf blade outlines, and smaller heads that lacked apical dominance and suppression of bracting. Notably, members of this subpopulation were also far more variable for many horticultural quality traits when compared with Calabrese broccoli, especially for head quality (first rank branching, bract suppression, head extension) and phenology traits (days to maturity and flowering), and lateral shoot formation. One-third of the sprouting broccoli accessions produced white flowers, a trait not observed in any other inferred subpopulation. The morphological diversity of these sprouting broccoli types is mirrored genetically; sprouting broccoli accessions were far more rich in unique polymorphic alleles per accession when compared to Calabrese landraces and hybrids. While the head morphology of some sprouting broccoli accessions (e.g.; B119 and B134) resembled the Calabrese broccoli ideotype, these accessions were clearly genetically resolved from Calabrese broccoli and may be an example of a parallel or convergent domestication syndrome within italica.

We did not observe clear evidence that sprouting broccoli is a direct recent progenitor of Calabrese broccoli or violet cauliflower. In fact, the genetic distance between sprouting broccoli and Calabrese broccoli is roughly the same as the distance between Calabrese broccoli and cauliflower ${ }^{16}$, raising the question of the placement of sprouting broccoli within $B$. oleracea. The distinctions between the Calabrese and sprouting broccoli types may be explained by reproductive isolation due to either geographic isolation (Sicily vs. the Italic Peninsula) or in-situ cultural practices isolating these groups as distinct crops. In a separate analysis that derived SNPs generated from alignment to a different $B$. oleracea reference genome ${ }^{8}$, the members of the sprouting broccoli subpopulation were differentiated into separate supopulations, and these groups were morphologically resolved by differences in head mass, head shape, and bracting suppression, indicative of further structure within the sprouting broccoli subpopulation. Interestingly, many of the sprouting broccoli accessions bear some morphological similarities to Chinese kale ( $B$. oleracea var. alboglabra), such as a weaker apical dominance, smaller, highly bracted heads with large flower buds, and variable white/yellow flower color, although this relationship cannot be evaluated here. Overall, the sprouting broccoli likely represents a valuable pool of genetic diversity that may prove useful for Calabrese improvement efforts as a source of diseaseresistance alleles or horticultural quality characteristics.

Collected landraces assigned to the violet cauliflower subpopulation were collected almost entirely in Sicily (except for B139.Di.Sicilia.Catanese, a likely import from Catania). The violet cauliflower population was similar to other botrytis types, overall exhibiting an earlier meristem arrest stage compared with sprouting and Calabrese broccoli accessions, although arrest stage ranged from floral primordia to fully developed flower buds. Under unfavorable environmental conditions, Calabrese broccoli hybrids often produce loose heads with irregular bud uniformity. In this evaluation, some violet cauliflower accessions consistently exhibited large, uniform, and compact heading structures and high bud uniformity, traits potentially useful in Calabrese broccoli breeding programs. It has been previously proposed that curding type botrytis arose from heading Calabrese broccoli via intermediate Sicilian types ${ }^{12,47}$. Evaluating the accessions observed in this study, it is unlikely that the Calabrese landraces formed the genetic basis of the violet cauliflower accessions. With the exception of recent intentional italica/botrytis hybrids, these subpopulations were clearly distinct and highly resolved by phylogenetic inference and structure analysis.

Our analysis supports several key findings: Modern $\mathrm{F}_{1}$ hybrid Calabrese broccoli has undergone strong selective pressures and reduction in diversity compared to the open-pollinated landraces it was derived from. Morphologically, modern $F_{1}$ hybrid Calabrese broccoli is distinct from its landrace predecessors, exhibiting accelerated maturity, more complete apical meristem arrest and dominance, higher harvest index, and superior heading quality characteristics. Several landrace accessions appear to be foundational as the initial source germplasm for modern hybrid Calabrese broccoli. While there are numerous signals of selection, several key genomic regions of reduced diversity and selective sweeps are particularly obvious with Calabrese broccoli hybrids and these regions harbor developmental candidates. Calabrese broccoli landraces are 4.8 -fold richer in allelic diversity compared to Calabrese hybrids, and the larger pool of italica germplasm is more far rich in allelic diversity than is captured in modern hybrid Calabrese broccoli, and this diversity must be preserved as a resource for future 
broccoli and cauliflower improvement. There is not clear evidence that sprouting broccoli or violet cauliflower are the direct progenitor of Calabrese broccoli, or vice versa.

This work provides an overview of the genetic and morphological diversity in B. oleracea var. italica and clarifies the relationship of modern Calabrese broccoli hybrids with foundational germplasm via analysis of morphological changes, population differentiation, allelic diversity, selective sweeps, linkage disequilibrium, runs of homozygosity, and key population-specific SNPs. These results quantify the genetic erosion occurring in italica and underscores the importance of in-situ and ex-situ conservation efforts.

\section{Methods}

\section{Genotyping}

Leaf tissue from all entries were bulked from five plants at the 2-3 true leaf stage and extracted according to standard protocols ${ }^{70}$. Genotyping-by-sequencing (GBS) was accomplished at the University of Wisconsin Biotechnology Center DNA Sequencing Facility as previously described $^{71}$. Libraries were construction in two 96-well plates using digestion by the restriction enzyme ApeKI and were sequenced on Illumina HiSeq 2500, producing 100bp single-end reads. SNPs were produced using the TASSEL v5.2.57 GBS pipeline ${ }^{72}$. The raw sequence reads were aligned to the B. oleracea BOL.v2 ${ }^{6}$ reference genome using BurrowsWheeler Alignment (v.0.7.17) backtrack algorithm ${ }^{73}$. The TASSEL commands -DiscoverySNPCallerPluginV2 and -ProductionSNPCallerV2 were invoked with default parameters, followed by LD-KNNi imputation ${ }^{74}$ with the following parameters: high LD sites (30), number of nearest neighbors (10), max distance to find LD $(10,000,000)$. Indels or sites assigned to unplaced scaffolds were removed, and one entry (B140) was removed from further analysis due to missing data, resulting in $N=109$ entries. Sites with $>10 \%$ missing data and minor allele frequencies $(\mathrm{MAF}<0.05)$ were removed, resulting in 31,811 SNPs. GBS summary statistics by site, chromosome, taxa, and subpopulation were generated in TASSEL and visualized in R v3.6.175. To identify subpopulation specific polymorphic alleles, the GBS data were divided by subpopulation for taxa assigned $50 \%$ membership to a given subpopulation in structure analysis and subsequently filtered for MAF $>5 \%$, minor SNP states, and indels. Genome-wide SNP density was calculated by binning markers in $1 \mathrm{Mbp}$ bins across physical locations. Linkage disequilibrium was calculated for all accessions and independently within subpopulations by estimating $\mathrm{r}^{2}$ for a given marker against all other markers within a $1 \mathrm{Mbp}$ window using vcftools ${ }^{76}$ by invoking the -geno-r2 command. To compare linkage disequilibrium between subpopulations, distances in base pairs were $\log _{10}$ transformed and fit using a smoothed spline $(\mathrm{spar}=0.5)$. Decay below the threshold $\left(r^{2}<0.2\right)$ was determined by choosing the smallest value of the smoothed spline falling below threshold values. For the Calabrese hybrids, the function estimate coefficients across all genomic regions were extracted and the top 1 percentile was selected. These regions were merged if overlap was less than $1 \mathrm{Mbp}$ and intersected with the BOL.v2 annotation using the R package bedr v1.0.7 ${ }^{77}$. Variant annotation was accomplished for all 31,811 markers using SnpEff v.4.3t $\mathrm{t}^{78}$ using the BOL.v2 annotation under default settings.

\section{Diversity}

PCA and multidimensional scaling using all markers and taxa was conducted in TASSEL by invoking the -PrincipalComponentsPlugin and-MultiDimensionalScalingPlugin, respectively. The PCA loadings explaining variance in PCA structure for each SNPs were calculated in TASSEL and the top $1 \%$ SNPs were filtered and intersected with the BOL.v2 annotation. Using all 31,811 markers and taxa $(N=109)$, a genetic similarity matrix was generated in PLINK (v1.90b6.15) ${ }^{79}$ by calculating the probability that randomly chosen alleles at a locus are identical by state $\left(\mathrm{P}_{\mathrm{IBS}}(\mathrm{AA}, \mathrm{AA})=1 ; \quad \mathrm{P}_{\mathrm{IBS}}(\mathrm{AA}, \mathrm{BB})=0, \quad \mathrm{P}_{\mathrm{IBS}}(\mathrm{AA}, \mathrm{xx})=0.5\right)$, averaged over all non-missing loci by invoking the command -distance with the 'square' and 'ibs' parameters. The similarity matrix was visualized using the heatmap2() function in R. A maximum likelihood based inference of phylogeny was conducted using the program RAxML (8.2.12) ${ }^{80,81}$ using the CIPRES Science Gateway server ${ }^{82}$ under the ML/thorough-bootstrap workflow, using the GTR + GAMMA bootstrapping model with one hundred alternative runs on distinct starting trees. The consensus tree was rooted using using the function $\operatorname{root}()$ in the $\mathrm{R}$ package ape ${ }^{83}$ and used to order the rows of the distance matrix and population structure analysis. For population structure analysis, the 31,811 SNPs were first pruned for linkage disequilibrium in PLINK using the function -indep-pairwise using a step size of $50 \mathrm{~kb}$, a window size of $1 \mathrm{~kb}$, and linkage disequilibrium threshold $\left(r^{2}>0.25\right)$. The Bayesian clustering algorithm structure.py was run across the values of $K 2.20$, assuming a simple prior in FastStruture (v.1.0) ${ }^{84}$. The FastStruture chooseK.py algorithm selected $K=4$ as the optimal model complexity to explain the structure within the data as well as maximizing the marginal likelihood. All accessions were assigned to a subpopulation with a minimum threshold of $50 \%$ membership. Structure results were visualized using the R package popHelper ${ }^{85}$.

Genome-wide population differentiation $\left(\mathrm{F}_{\mathrm{st}}\right)$ was calculated when comparing the Calabrese hybrid subpopulation against all other subpopulations, using implementing the -weir-fst-pop function in vcftools to scan across all 31,811 markers in $1 \mathrm{Mbp}$ windows with a $1 \mathrm{kbp}$ step-size. Nucleotide diversity was estimated genome-wide by the sliding-window TASSEL plugin -diversitySlidingWinStep across 10 SNP markers using a 5 
marker step size. Nucleotide diversity and reduced/enriched diversity were calculated as where $\mathrm{x}_{\mathrm{i}}$ is the frequency of the ith sequence in the population and $\mathrm{pi}_{j}$ is the number of differences per nucleotide site between the ith and jth sequences ${ }^{86}$. ROD was calculated across 10 markers using a 5 marker step-width and the top 1 percentile genomic regions ${ }^{5,87,88}$ were reserved for further analysis. Selective sweep analysis was conducting using the Calabrese hybrid subpopulation with SweeD ${ }^{89}$. SweeD was run for each subpopulation by first disaggregating all 31,811 markers by chromosome and then adjusting the grid size to scan $1 \mathrm{kbp}$ windows of each chromosome using a custom bash script. A screen for clustered runs of homozygousity were was conducted in PLINK using the -homozyg-group algorithm to scan across $1 \mathrm{Mbp}$ windows, requiring a 0.99 or greater segment concordance between pairwise matches, allowing for one heterozygous call and five missing calls within windows. The top 1 percentile outliers for these regions were intersected with the BOLv.2 annotation using and a custom $\mathrm{R}$ script and the $\mathrm{R}$ package bedr that merged adjacent regions separated by less than $1 \mathrm{Mbp}$. These feature-rich genomic regions were searched against the online databases Ensembl Plants using the $\mathrm{R}$ package biomaRt $\mathrm{t}^{90,91}$ and Tair $^{92,93}$ and visualized using a custom $\mathrm{R}$ script.

\section{Germplasm and phenotyping}

Hybrid $F_{1}$ germplasm was gathered from multiple breeding programs and distributors (Supplementary Table 1). Landrace accessions collected in Italy typically contained geographical provenance, but if exact latitude and longitude coordinates were not supplied (e.g., an address), latitude and longitude was determined using Google Maps and mapped using the package maps ${ }^{94}$. Additional passport information including cultivar release year and breeding institution was collected from several sources $^{16,51,95}$, and personal communication with breeders.

Lines were sown into 128 cell trays on 2019/05/05 $(J D=125)$ and 2019/06/03 (JD = 154) for plantings 1 and 2 . Seedlings were grown in a greenhouse, transferred to cold frames after 4 weeks, and transplanted on JD 170 and JD 189 respectively into Lima silt loam fields in Geneva, NY $(42.88 \mathrm{~N},-77.03 \mathrm{~W})$. All lines were randomized into three replications and transplanted onto raised beds with $\sim 10$ plants per genotype per plot, although some plots contained fewer plants due to seed low seed quantity or poor germination. Drip irrigation was applied as needed throughout the growing season and additional cultural practices were as previously described ${ }^{28,52}$. Plots were examined every other day and evaluated at heading maturity for heading traits, determined when between $1 / 3$ to $2 / 3$ of plants in a given plot had reached harvest stage.

Approximately 16 biennial or day length sensitive accessions that would normally form heading structures in cooler season Mediterranean climates did not head or flower and were excluded from evaluations. Traits within four trait classes were evaluated: architecture, biomass, head quality, and phenology ${ }^{27}$ (Supplementary Table 1 TRAITS). The traits (MT, HE, BR, BS, BU, HC, HU, and OQ) followed standardized protocol employed by the Eastern Broccoli Project ${ }^{52}$ using an ordinal scale $(1=$ worst; $5=$ best). Plants were cut at ground level to evaluate BM, HT, WD, LT. Heads were trimmed $16 \mathrm{~cm}$ from crown apex, photographed, processed using the GNU Image Manipulation Program v2.10.8 ${ }^{96}$ and visualized using a custom R script. The traits (HT, WD, LT, BM, HM, FC, FF, F50) were evaluated according to IBPGR standards ${ }^{97}$. The traits HD, $\mathrm{CL}$, and RR were collected according to previously described protocols ${ }^{98}$. The phenology traits (MT, FF, F50) were calculated as days from sowing to maturity, first flowering, and 50\% flowering respectively; holding ability (MT50) was calculated as F50 - MT. Summary statistics were calculated by summarizing genotypes across traits and environments. Spearman correlation coefficients were estimated using complete pairwise observations. Linear regression was conducted in $\mathrm{R}$ to compare horticultural quality traits with cultivar release year of relevant accessions and principal component axes. Relative importance analysis of horticultural quality was conducted using 1000 bootstrap replications of the "lmg" method in the $\mathrm{R}$ package RateRvaR (v. 1.0) ${ }^{52,99}$ using nonaveraged data by fitting the model: $\mathrm{OQ} \sim \mathrm{HT}+\mathrm{WD}+\mathrm{HE}+\mathrm{LT}+\mathrm{BM}+\mathrm{HM}+$ $\mathrm{BS}+\mathrm{BU}+\mathrm{HC}+\mathrm{HD}+\mathrm{CL}+\mathrm{RR}+\mathrm{BR}+\mathrm{HS}+\mathrm{HU}+\mathrm{HR}$ $+\mathrm{MT}+\mathrm{FF}+\mathrm{F} 50$.

\section{Acknowledgements}

We thank Colden Proe and Teagan Zingg for assistance with phenotyping and image processing. Sandra Branham, Mackenzie Mabry, and Joanne Labate provided valuable manuscript feedback. Ferdinando Branca, Phillip Griffiths, and Mark Farnham provided additional seed. This work is supported by Specialty Crop Research Initiative grant no. 2016-51181-25402 from the USDA National Institute of Food and Agriculture.

\section{Author contributions}

Z.S. conducted phenotyping and statistical analyses. All authors conducted experimental design and wrote the manuscript.

\section{Data availability}

All data is included in supplementary materials or SRA:SUB7486659.

\section{Code availability}

All code used in this analysis is available at https://github.com/zacharystansell/ B_oleracea_diversity_panel.

Conflict of interest

The authors declare that they have no conflict of interest.

Supplementary Information accompanies this paper at (https://doi.org/ 10.1038/s41438-020-00375-0).

Received: 22 May 2020 Revised: 12 July 2020 Accepted: 1 August 2020 Published online: 01 October 2020 


\section{References}

1. Food and Agriculture Organization of the United Nations. FAOSTAT Statistical Database (1997). http://www.fao.org/faostat/en/. March 2020.

2. USDA-NASS. NASS Vegetables Survey (2017) https://www.nass.usda.gov/ Surveys/Guide_to_NASS_Surveys/Negetables. March 2020.

3. Allender, C. J., Allainguillaume, J., Lynn, J. \& King, G. J. Simple sequence repeats reveal uneven distribution of genetic diversity in chloroplast genomes of Brassica oleracea L. and ( $n=9$ ) wild relatives. Theor. Appl. Genet. 114, 609-618 (2007).

4. Cheng, F. et al. Genome resequencing and comparative variome analysis in a Brassica rapa and Brassica oleracea collection. Sci. Data 3, 1-9 (2016).

5. Cheng, F. et al. Subgenome parallel selection is associated with morphotype diversification and convergent crop domestica-tion in Brassica rapa and Brassica oleracea. Nat. Genet. 48, 1218 (2016).

6. Parkin, I. A. et al. Transcriptome and methylome profiling reveals relics of genome dominance in the mesopolyploid Brassica oleracea. Genome Biol. 15, R77 (2014).

7. Golicz, A. A. et al. The pangenome of an agronomically important crop plant Brassica oleracea. Nat. Commun. 7, 13390 (2016)

8. Belser, C. et al. Chromosome-scale assemblies of plant genomes using nanopore long reads and optical maps. Nat. Plants 4, 879 (2018).

9. Lázaro, A. \& Aguinagalde, I. Genetic diversity in Brassica oleracea L. (Cruciferae) and wild relatives $(2 n=18)$ using isozymes. Ann. Bot. $\mathbf{8 2}$ 821-828 (1998).

10. Lowman, A. C. \& Purugganan, M. D. Duplication of the Brassica oleracea APETALA1floral homeotic gene and the evolution of domesticated cauliflower. J. Hered. 90, 514-520 (1999)

11. Purugganan, M. D., Boyles, A. L. \& Suddith, J. I. Variation and Selection at the CAULIFLOWER Floral Homeotic Gene Accompanying the Evolution of Domesticated Brassica oleracea. Genetics 155, 855-862 (2000).

12. King, G. J. Using molecular allelic variation to understand domestication processes and conserve diversity in Brassica crops. In International Symposium on Sustainable Use of Plant Biodiversity to Promote New Opportunities for Horticultural Production 598, 181-186 (2003).

13. Maggioni, L., von Bothmer, R., Poulsen, G. \& Branca, F. Origin and domestication of cole crops (Brassica oleracea L.): linguistic and literary considerations1. Econ. Bot. 64, 109-123 (2010).

14. Ciancaleoni, S., Chiarenza, G. L., Raggi, L., Branca, F. \& Negri, V. Diversity characterisation of broccoli (Brassica oleracea L. var. italica Plenck) landraces for their on-farm (in situ) safeguard and use in breeding programs. Genet. Resour. Crop. Evol. 61, 451-464 (2014).

15. Yousef, E. A. A. Müller, T., Börner, A. \& Schmid, K. J. Comparative analysis of genetic diversity and differentiation of cauliflower (Brassica oleracea var. botrytis) accessions from two ex situ genebanks. PLOS ONE 13, e0192062 (2018).

16. Stansell, Z. et al. Genotyping-by-sequencing of Brassica oleracea vegetables reveals unique phylogenetic patterns, population structure and domestication footprints. Hortic. Res. 5, 38 (2018).

17. Branca, F. et al. Diversity of Sicilian broccoli (Brassica oleracea var. italica) and cauliflower (Brassica oleracea var. botrytis) landraces and their distinctive biomorphological, antioxidant, and genetic traits. Genet. Resour. Crop. Evol. 65 485-502 (2018)

18. Edger, P. P. et al. Brassicales phylogeny inferred from 72 plastid genes: a reanalysis of the phylogenetic localization of two paleopolyploid events and origin of novel chemical defenses. Am. J. Bot. 463-469, https://doi.org/ 10.1002/ajb2.1040@10.1002.Tree-of-Life-virtual-issue (2018).

19. Maggioni, L., von Bothmer, R., Poulsen, G. \& Lipman, E. Domestication, diversity and use of Brassica oleracea L., based on ancient Greek and Latin texts. Genet Resour. Crop. Evol. 65, 137-159 (2018).

20. Li, Z. et al. The evolution of genetic diversity of broccoli cultivars in China since 1980. Sci. Hortic. 250, 69-80 (2019).

21. Mabry, M. E. et al. Phylogeny and multiple independent whole-genome duplication events in the Brassicales. bioRxiv 789040, https://doi.org/10.1101/ 789040 (2019).

22. Walley, P. G. et al. Developing genetic resources for pre-breeding in Brassica oleracea L:: an overview of the UK perspective. J. Plant Biotechnol. 39, 62-68 (2012).

23. Walley, P. G. et al. A new broccoli $x$ broccoli immortal mapping population and framework genetic map: tools for breeders and complex trait analysis. Theor. Appl. Genet. 124, 467-484 (2012).
24. LV, H. et al. Whole-Genome Mapping Reveals Novel QTL Clusters Associated with Main Agronomic Traits of Cabbage (Brassica oleracea var. capitata L.). Front. Plant Sci. 7, https://doi.org/10.3389/fpls.2016.00989 (2016).

25. Li, $\mathrm{H}$. et al. Curd development associated gene (CDAG1) in cauliflower (Brassica oleracea L. var. botrytis) could result in enlarged organ size and increased biomass. Plant Sci. 254, 82-94 (2017).

26. Sun, $X$. et al. Effect of ambient temperature fluctuation on the timing of the transition to the generative stage in cauliflower. Environ. Exp. Bot. 155, 742-750 (2018)

27. Stansell, Z., Farnham, M. \& Björkman, T. Complex horticultural quality traits in broccoli are illuminated by evaluation of the immortal BolTBDH mapping population. Front. Plant Sci. 10, 1104 (2019).

28. Farnham, M. W. \& Björkman, T. Evaluation of Experimental Broccoli Hybrids Developed for Summer Production in the Eastern United States. HortScience 46, 858-863 (2011)

29. Matschegewski, C. et al. Genetic variation of temperature-regulated curd induction in cauliflower: elucidation of floral transition by genome-wide association mapping and gene expression analysis. Front. Plant Sci. 6, 720 (2015).

30. Irwin, J. A. et al. Nucleotide polymorphism affecting FLC expression underpins heading date variation in horticultural brassicas. Plant J. 87, 597-605 (2016).

31. Branham, S. E., Stansell, Z. J., Couillard, D. M. \& Farnham, M. W. Quantitative trait loci mapping of heat tolerance in broccoli (Brassica oleracea var. italica) using genotyping-by-sequencing. Theor. Appl. Genet. 130, 529-538 (2017).

32. Shea, D. J. et al. The role of FLOWERING LOCUS C in vernalization of Brassica: the importance of vernalization research in the face of climate change. Crop. Pasture Sci. 69, 30-39 (2018).

33. Branham, S. E. \& Farnham, M. W. Identification of heat tolerance loci in broccoli through bulked segregant analysis using whole genome resequencing. Euphytica 215, 34 (2019)

34. Zhu, X., Tai, X., Ren, Y., Chen, J. \& Bo, T. Genome-wide analysis of coding and long non-coding RNAs involved in cuticular wax biosynthesis in Cabbage (Brassica oleracea L. var. capitata). Int. J. Mol. Sci. 20, 2820, https://doi.org/ 10.3390/ijms20112820 (2019). Publisher: Multidisciplinary Digital Publishing Institute.

35. Lin, C.-W. et al. Analysis of ambient temperature-responsive transcriptome in shoot apical meristem of heat-tolerant and heat-sensitive broccoli inbred lines during floral head formation. BMC Plant Biol. 19, 3 (2019).

36. Gray, A. R. Taxonomy and evolution of broccoli (Brassica oleracea var. italica). Econ. Bot. 36, 397-410 (1982).

37. dos Santos, J. B., Nienhuis, J., Skroch, P., Tivang, J. \& Slocum, M. K. Comparison of RAPD and RFLP genetic markers in determining genetic similarity among Brassica oleracea L. genotypes. Theor. Appl. Genet. 87, 909-915 (1994).

38. Branca, F., Li, G., Goyal, S. \& Quiros, C. F. Survey of aliphatic glucosinolates in Sicilian wild and cultivated Brassicaceae. Phytochemistry 59, 717-724 (2002).

39. Prohens-Tomás, J. \& Nuez, F. Vegetables I: Asteraceae, Brassicaceae, Chenopodicaceae, and Cucurbitaceae (Springer Science \& Business Media, 2007).

40. Ciancaleoni, S., Raggi, L. \& Negri, V. Genetic outcomes from a farmer-assisted landrace selection programme to develop a synthetic variety of broccoli. Plant Genet. Resour. 12, 349-352 (2014).

41. Torricelli, R., Ciancaleoni, S. \& Negri, V. Performance and stability of homogeneous and heterogeneous broccoli (Brassica oleracea L. var. italica Plenck) varieties in organic and low-input conditions. Euphytica 199, 385-395 (2014).

42. Nicoletto, C., Santagata, S., Pino, S. \& Sambo, P. Caracterização antioxidante de diferentes variedades crioulas de brócolis italiano. Hortic. Brasileira 34, 74-79 (2016).

43. Tribulato, A., Donzella, E., Sdouga, D., Lopes, V. \& Branca, F. Bio-morphological characterization of Mediterranean wild and cultivated Brassica species. Acta Hortic. 1, 9-16 (2018).

44. Hammer, K, Montesano, V., Direnzo, P. \& Laghetti, G. Conservation of Crop Genetic Resources in Italy with a Focus on Vegetables and a Case Study of a Neglected Race of Brassica Oleracea. Agriculture 8, 105 (2018).

45. Snogerup, S. The wild forms of the Brassica oleracea group $(2 n=18)$ and their possible relations to the cultivated ones. (Jap. Sci. Soc. Press, Tokyo, 1980).

46. Kianian, S. F. \& Quiros, C. F. Generation of a Brassica oleracea composite RFLP map: linkage arrangements among various populations and evolutionary implications. Theor. Appl. Genet. 84, 544-554 (1992).

47. Massie, I. H., Astley, D. \& King, G. J. Patterns of genetic diversity and relationships between regional groups and populations of italian landrace cauliflower and broccoli (Brassica oleracea L. var. botrytis I. and var. italica plenck. Acta Hortic. 407, 45-54 (1996). 
48. Gómez-Campo, C. \& Prakash, S. Origin and domestication. In Gómez-Campo, C. (ed.) Developments in Plant Genetics and Breeding, vol. 4 of Biology of Brassica Coenospecies, 33-58, https://doi.org/10.1016/S0168-7972(99)80003-6 (Elsevier, 1999).

49. Vavilov, N. The origin, variation, immunity and breeding of cultivated plants, vol. 72 (LWW, 1951), chronica botanica edn

50. Buck, P. A. Origin and taxonomy of broccoli. Econ. Bot. 10, 250-253 (1956).

51. Farnham, M. W., Keinath, A. P. \& Grusak, M. A. Mineral concentration of broccoli florets in relation to year of cultivar release. Crop. Sci. 51, 2721-2727 (2011).

52. Stansell, Z., Björkman, T., Branham, S., Couillard, D. \& Farnham, M. W. Use of a quality trait index to increase the reliability of phenotypic evaluations in Broccoli. HortScience 52, 1490-1495 (2017).

53. Balkaya, A. \& Yanmaz, R. Promising kale (Brassica oleracea var. acephala) populations from Black Sea region, Turkey. New Zealand. J. Crop. Hortic. Sci. 33, 1-7 (2005).

54. Farnham, M. W., Davis, E. H., Morgan, J. T. \& Smith, J. P. Neglected landraces of collard (Brassica oleracea L. var. viridis) from the Carolinas (USA). Genet. Resour. Crop. Evol. 55, 797-801 (2008).

55. Pelc, S. E., Couillard, D. M., Stansell, Z. J. \& Farnham, M. W. Genetic diversity and population structure of collard landraces and their relationship to other Brassica oleracea Crops. The Plant Genome 8, https://doi.org/10.3835/ plantgenome2015.04.0023 (2015).

56. Stansell, Z., Cory, W., Couillard, D. \& Farnham, M. Collard landraces are novel sources of glucoraphanin and other aliphatic glucosinolates. Plant Breed. 134 350-355 (2015).

57. Branca, F. \& lapichino, G. Some wild and cultivated Brassicaceae exploited in Sicily as vegetables. Plant Genet. Resour. Newsl. (IPGRI/FAO) Bull. des Ressources Phytogenet. (IPGRI/FAO) Noticiario de Recursos Fitogeneticos (IPGRI/FAO) (1997)

58. Laghetti, G. et al. "Mugnoli": a neglected race of Brassica oleracea L. from Salento (Italy). Genet. Resour. Crop. Evol. 52, 635-639 (2005).

59. Hammer, K., Gladis, T., Laghetti, G. \& Pignone, D. The wild and the grown remarks on Brassica. In The wild and the grown - remarks on Brassica, https:// doi.org/10.17660/ActaHortic.2013.1005.2 (2013).

60. Björkman, T. \& Pearson, K. J. High temperature arrest of inflorescence development in broccoli (Brassica oleracea var. italica L.). J. Exp. Bot. 49, 101-106 (1998).

61. Duclos, D. V. \& Björkman, T. Meristem identity gene expression during curd proliferation and flower initiation in Brassica oleracea. J. Exp. Bot. 59, 421-433 (2008).

62. Wang, J. et al. Genome-wide nucleotide patterns and potential mechanisms of genome divergence following domestication in maize and soybean. Genome Biol. 20, 74 (2019).

63. Ceballos, F. C., Joshi, P. K., Clark, D. W., Ramsay, M. \& Wilson, J. F. Runs of homozygosity: windows into population history and trait architecture. Nat. Rev. Genet. 19, 220-234 (2018).

64. Lagercrantz, U. \& Lydiate, D. J. Comparative genome mapping in Brassica. Genetics 144, 1903-1910 (1996).

65. Okazaki, K et al. Mapping and characterization of FLC homologs and QTL analysis of flowering time in Brassica oleracea. Theor. Appl. Genet. 114, 595-608 (2007).

66. Uptmoor, R., Schrag, T., Stützel, H. \& Esch, E. Crop model based QTL analysis across environments and QTL based estimation of time to floral induction and flowering in Brassica oleracea. Mol. Breed. 21, 205-216 (2008).

67. Razi, H., Howell, E. C., Newbury, H. J. \& Kearsey, M. J. Does sequence polymorphism of FLC paralogues underlie flowering time QTL in Brassica oleracea?. Theor. Appl. Genet. 116, 179-192 (2008).

68. Hasan, Y. et al. Quantitative trait loci controlling leaf appearance and curd initiation of cauliflower in relation to temperature.Theor. Appl. Genet. 129 1273-1288 (2016).

69. Branham, S. E. \& Farnham, M. W. Genotyping-by-sequencing of waxy and glossy near-isogenic broccoli lines. Euphytica 213, 84 (2017).

70. Doyle, J. J. A rapid DNA isolation procedure for small quantities of fresh leaf tissue. Phytochem. Bull. 19, 11-15 (1987).

71. Elshire, R. J. et al. A robust, simple genotyping-by-sequencing (GBS) approach for high diversity species. PLOS ONE 6, e19379 (2011).
72. Glaubitz, J. C. et al. TASSEL-GBS: a high capacity genotyping by sequencing analysis pipeline. PLOS ONE 9, e90346 (2014).

73. $\mathrm{Li}, \mathrm{H}$. Aligning sequence reads, clone sequences and assembly contigs with BWA-MEM. arXiv:1303.3997 [q-bio] (2013)

74. Money, D. et al. Linklmpute: fast and accurate genotype imputation for nonmodel organisms. G3: Genes Genomes Genet. 5, 2383-2390 (2015).

75. R Core Team. R: A Language and Environment for Statistical Computing (2019).

76. Danecek, P. et al. The variant call format and VCFtools. Bioinformatics 27, 2156-2158 (2011).

77. Haider, S. et al. A bedr way of genomic interval processing. Source Code for Biol. Medicine 11, 14 (2016).

78. Cingolani, P. et al. A program for annotating and predicting the effects of single nucleotide polymorphisms, SnpEff: SNPs in the genome of Drosophila melanogaster strain w1118; iso-2; iso-3. Fly 6, 80-92 (2012).

79. Purcell, S. et al. PLINK: a tool set for whole-genome association and population-based linkage analyses. The Am. J. Hum. Genet. 81, 559-575 (2007).

80. Stamatakis, A. RAxML version 8: a tool for phylogenetic analysis and postanalysis of large phylogenies. Bioinformatics 30, 1312-1313 (2014).

81. Bird, K. A. et al. Population Structure and Phylogenetic Relationships in a Diverse Panel of Brassica rapa L. Front. Plant Sci. 8, https://doi.org/10.3389/ fpls.2017.00321 (2017).

82. Miller, M. A., Pfeiffer, W. \& Schwartz, T. Creating the CIPRES Science Gateway for inference of large phylogenetic trees. In 2010 Gateway Computing Environments Workshop (GCE), 1-8, https://doi.org/10.1109/GCE.2010.5676129 (2010).

83. Paradis, E., Claude, J. \& Strimmer, K. APE: analyses of Phylogenetics and Evolution in R language. Bioinformatics 20, 289-290 (2004).

84. Raj, A., Stephens, M. \& Pritchard J.K.fastSTRUCTURE: variational inference of population structure in large SNP data sets. Genetics 197, 573-589 (2014).

85. Francis, R.M. pophelper: an R package and web app to analyse and visualize population structure. Mol. Ecol. Resour. 17, 27-32 (2017).

86. Nei, M. \& Li, W. H. Mathematical model for studying genetic variation in terms of restriction endonucleases. Proc. Natl Acad. Sci. USA. 76, 5269-5273 (1979).

87. Tajima, F. Evolutionary relationship of DNA sequences in finite populations. Genetics 105, 437-460 (1983).

88. $\mathrm{Xu}, \mathrm{X}$. et al. Resequencing 50 accessions of cultivated and wild rice yields markers for identifying agronomically important genes. Nat. Biotechnol. 30, 105-111 (2012).

89. Pavlidis, P., Živkovic, D., Stamatakis, A. \& Alachiotis, N. SweeD: likelihood-based detection of selective sweeps in thousands of genomes. Mol. Biol. Evol. 30 2224-2234 (2013).

90. Durinck, S. et al. BioMart and bioconductor: a powerful link between biological databases and microarray data analysis. Bioinformatics 21, 3439-3440, https:// doi.org/10.1093/bioinformatics/bti525 (2005). Publisher: Oxford Academic.

91. Durinck, S., Spellman, P. T., Birney, E. \& Huber, W. Mapping identifiers for the integration of genomic datasets with the R/bioconductor package biomaRt. Nat. Protoc. 4, 1184-1191 (2009).

92. Berardini, T. Z. et al. The arabidopsis information resource: making and mining the "gold standard" annotated reference plant genome. genesis 53, 474-485 (2015).

93. Ruffier, M. et al. Ensembl core software resources: storage and programmatic access for DNA sequence and genome annotation. Database 2017, https://doi. org/10.1093/database/bax020 (2017).

94. Richard A. Becker, Allan R. Wilks, Ray Brownrigg, Thomas P. Minka \& Alex Deckmyn. maps: Draw Geographical Maps (2018).

95. Mark Farnham. Vegetable Cultivar Descriptions for North America - Broccoli | Cucurbit Breeding.

96. The GIMP Development Team. GIMP (2019).

97. International Board for Plant Genetic Resources. Descriptors for Brassica and Raphanus (International Board for Plant Genetic Resources, Rome, 1990).

98. Lan, T. H. \& Paterson, A. H. Comparative mapping of quantitative trait loci sculpting the curd of Brassica oleracea. Genetics 155, 1927-1954 (2000).

99. Stansell, Z. J., Akdemir, D. \& Björkman, T. RateRvaR (2019). 